\section{Management of Sarcoidosis-associated Pulmonary Hypertension}

Editor - We read with interest Patel and Ladak's report ${ }^{1}$ of a patient presenting with tattoo koebnerization and severe pulmonary hypertension $(\mathrm{PH})$ in the setting of a new diagnosis of sarcoidosis. As the authors note, sarcoidosis-associated pulmonary hypertension (SAPH) is classified as World Health Organization group $5 \mathrm{PH}$, a "miscellaneous" form of $\mathrm{PH}$ that develops through various mechanistic pathways. ${ }^{2}$ The case patient was treated with vasodilators as an inpatient, improved, and then was discharged on a regimen that included ambrisentan and tadalafil for the treatment of SAPH.

Ambrisentan and tadalafil are not indicated for the treatment of SAPH. Endothelin receptor antagonists and phosphodiesterase-5 inhibitors are approved by the U.S. Food and Drug Administration (FDA) for the treatment of group $1 \mathrm{PH}$ only. No therapeutics have been approved by the FDA for the treatment of SAPH. The use of ambrisentan and tadalafil to treat SAPH represents offlabel use of these drugs, which may be appropriate for selected patients, as it apparently was for the case patient.

$\mathrm{PH}$ attributable to sarcoidosis appears to occur in a heterogeneous group of patients and is caused by a diversity of mechanisms. ${ }^{3}$ Both pre-capillary and post-capillary vascular pathology may result in the development of $\mathrm{PH}$ in patients with sarcoidosis. Both benefits and harms have been reported in patients with SAPH after initiation of pulmonary vasodilators. ${ }^{4}$ Among sarcoidosis patients with advanced fibrocavitary disease, PH-directed therapies may worsen oxygenation by inhibiting hypoxic pulmonary vasoconstriction, leading to increased shunting. ${ }^{5}$ Among SAPH patients with pulmonary venous disease, PH-directed treatment may result in pulmonary edema. ${ }^{6}$ Some studies have shown benefit from $\mathrm{PH}$-directed therapy for SAPH, supporting its use in well-selected patients. ${ }^{7}$

Management of SAPH in the outpatient setting with PH-directed medication requires close follow-up to assess for possible harms resulting from the drugs. Under most circumstances, it is advisable to treat SAPH by treating the underlying sarcoidosis that resulted in $\mathrm{PH}$, in addition to considering $\mathrm{PH}$-directed treatment. The cornerstone treatment for sarcoidosis is corticosteroids, but other anti-inflammatory and immunomodulatory therapies may be included. If PH-directed therapy is being considered for a patient with SAPH, the patient should be informed that there are no FDA-approved medications for SAPH and counseled about the potential harms that might result from $\mathrm{PH}$-directed treatment. Additional research is needed to define the precise role of anti-inflammatory and immunomodulatory therapeutics in the treatment of SAPH.

Keywords: Pulmonary hypertension; Sarcoidosis; Treatment; Management

\section{References}

1. Patel M, Ladak K. Tattoo koebnerization and severe pulmonary hypertension. Clin Med Res. 2019;17(3-4):105-106.

2. Diaz-Guzman E, Farver C, Parambil J, Culver DA. Pulmonary hypertension caused by sarcoidosis. Clin Chest Med. 2008;29(3):549-563, x.

3. Shlobin O, Baughman R. Sarcoidosis-associated pulmonary hypertension. Semin Respir Crit Care Med. 2017;38(04):450 462.

4. Huitema MP, Grutters JC, Rensing BJWM, Reesink HJ, Post MC. Pulmonary hypertension complicating pulmonary sarcoidosis. Neth Heart J. 2016;24(6):390-399.

5. Valeyre D, Nunes H, Bernaudin JF. Advanced pulmonary sarcoidosis. Curr Opin Pulm Med. 2014;20(5):488-495.

6. daSilva-deAbreu A, Mandras SA. Sarcoidosis-associated pulmonary hypertension: an updated review and discussion of the clinical conundrum. Curr Probl Cardiol. 2019;100506:100506; Epub ahead of print.

7. Dobarro D, Schreiber BE, Handler C, Beynon H, Denton CP, Coghlan JG. Clinical characteristics, haemodynamics and treatment of pulmonary hypertension in sarcoidosis in a single centre, and meta-analysis of the published data. Am J Cardiol. 2013;111(2):278-285.

Jim Q. Ho, BA

Albert Einstein College of Medicine, Bronx, New York; Email:jim.ho@einsteinmed.org

Ware G. Kuschner, MD

Pulmonary and Critical Care Medicine Section, Veterans Affairs Palo Alto Health Care System, Palo Alto, California; and Division of Pulmonary and Critical Care Medicine, Department of Medicine, Stanford University School of Medicine, Stanford, California

Received: January 31, 2020

Revised: May 14, 2020

Accepted: May 15, 2020

doi: $10.3|2| / \mathrm{cmr} .2020 .1556$

\section{Author Response: \\ Pulmonary Vasodilators in Sarcoidosis-associated Pulmonary Hypertension}

Editor - We thank Ho and Kuschner ${ }^{1}$ for their knowledgeable comments on our case, specifically around the need to individualize therapy for each patient, multimodal therapeutic interventions including immunosuppression, and close follow-up.

Sarcoidosis-associated pulmonary hypertension (SAPH) is often classified as WHO group 5.,3 However, the pathophysiology of pulmonary hypertension in sarcoidosis is multifactorial and it can also be classified as WHO group 1 (pulmonary arterial hypertension), group 2 (pulmonary hypertension from left heart disease), group 3 (pulmonary 
hypertension due to lung disease and/or hypoxaemia) or group 4 (pulmonary hypertension due to chronic thromboembolic pulmonary hypertension). ${ }^{4}$ As the etiology of pulmonary hypertension in sarcoidosis is often multifactorial, the use of vasodilators may prove beneficial in well-selected patients, resulting in improved functional capacity, quality of life and hemodynamics despite its off-label use. ${ }^{4}$

The treatment of SAPH has not been studied extensively and the best management strategy is currently unknown. ${ }^{3,4} \mathrm{~A}$ multidisciplinary approach, including cardiologists, respirologists and radiologists specializing in pulmonary hypertension should be used when treating such patients. ${ }^{4}$ As Ho and Kuschner ${ }^{1}$ point out, the cornerstone treatment of sarcoidosis includes immunosuppressive therapies such as corticosteroids; however, these medications are not always effective in isolation in treating $\mathrm{SAPH}$, and long-term use is associated with several unwanted consequences., ${ }^{5,6}$ The treatment for SAPH therefore can include pulmonary vasodilators, anticoagulants and supplemental oxygen. ${ }^{3}$

As an example, ambrisentan treats SAPH by blocking endothelin, a vasoconstrictive peptide involved in the development of pulmonary hypertension. ${ }^{3}$ Endothelin itself is found in higher concentrations in urine, plasma and bronchoalveolar lavage samples from patients with sarcoidosis., ${ }^{3,5}$ Phosphodiesterase-5 inhibitors such as tadalafil increase nitric oxide in the pulmonary arterial smooth muscle leading to vasodilation reducing pulmonary hypertension. ${ }^{5}$ The effectiveness of pulmonary vasodilators in the management of SAPH has been demonstrated by numerous studies, which found improved hemodynamics including lower mean pulmonary arterial pressures, pulmonary vascular resistance and brain natriuretic peptide as well as increased cardiac output. $^{7-15}$ Pulmonary vasodilators have also been associated with increased functionality demonstrated by improved 6-minute walking distances, New York Heart association class and the Borg dyspnea index in patients with sarcoidosis-

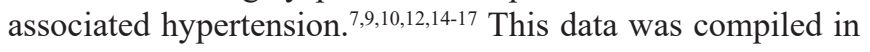
a meta-analysis which confirmed the beneficial aspects of pulmonary vasodilators in patients with SAPH. ${ }^{2}$

Ho and Kuschner ${ }^{1}$ note that pulmonary vasodilators may worsen oxygenation in patients with SAPH due to increased shunting. ${ }^{5}$ However, the use of pulmonary vasodilators, such as phosphodiesterase-5 inhibitors and endothelin receptor antagonists, in patients with SAPH has not been associated with worse outcomes such as increased mortality or the need for lung transplantation. ${ }^{2}$ Tadalafil has been used in SAPH with no reported major adverse events or clinical worsening. ${ }^{6}$ Ambrisentan is generally well tolerated and any adverse effects are mostly of mild to moderate intensity and resolve with discontinuation of the medication. ${ }^{16}$ Other studies in patients with severe parenchymal disease from sarcoidosis and associated SAPH reported no deterioration of oxygen saturation after administration of pulmonary vasodilators., 3
In order to ensure the safe use of pulmonary vasodilators in patients with sarcoidosis-associated pulmonary hypertension, candidates for these medications should be selected carefully, with particular caution if significant hypoxia or parenchymal disease exists, with follow-ups undertaken regularly. The broad range of responses to these medications reflects the complicated pathophysiologic nature of sarcoidosis and emphasizes the importance of individualizing care to each patient's presentation and prognosis. ${ }^{3,4}$

The lack of widespread evidence in support of these drugs may be in relation to small sample sizes of these studies and inappropriate inclusion criteria. ${ }^{5}$ Specifically, patients at the terminal stage of their disease or those with mild pulmonary vascular dysfunction are least likely to demonstrate a response to therapy. ${ }^{5}$

There is a clear need for further research into the management of SAPH as current reports are insufficient to guide widespread practice but do demonstrate the efficacy of pulmonary vasodilators in select patients. ${ }^{3}$ The rationale for the use of tadalafil and ambrisentan was explained to our patient and informed consent was obtained prior to the administration of these drugs. The patient experienced rapid recovery on the reported regimen of tadalafil, ambrisentan, methylprednisolone and adalimumab and at follow up reported no adverse effects from the medication in addition to continued disease remission.

Keywords: Sarcoidosis; Pulmonary hypertension; Pulmonary vasodilators

\section{References}

1. Ho JQ, Kuschner WG. Management of sarcoidosis-associated pulmonary hypertension. Clin Med Res. 2020;2-3:1.

2. Dobarro D, Schreiber BE, Handler C, Beynon H, Denton CP, Coghlan JG. Clinical characteristics, haemodynamics and treatment of pulmonary hypertension in sarcoidosis in a single centre, and meta-analysis of the published data. Am J Cardiol. 2013;111(2):278-285.

3. Diaz-Guzman E, Farver C, Parambil J, Culver DA. Pulmonary hypertension caused by sarcoidosis. Clin Chest Med. 2008;29(3):549-563.

4. Huitema MP, Grutters JC, Rensing BJWM, Reesink HJ, Post MC. Pulmonary hypertension complicating pulmonary sarcoidosis. Neth Heart J. 2016;24(6):390-399.

5. daSilva-deAbreu A, Mandras SA. Sarcoidosis-associated pulmonary hypertension: an updated review and discussion of the clinical conundrum [published online ahead of print, 2019 Nov 30]. Curr Probl Cardiol. 2019;100506.

6. Ford HJ, Baughman RP, Aris R, Engel P, Donohue JF. Tadalafil therapy for sarcoidosis-associated pulmonary hypertension. Pulm Circ. 2016;6(4):557-562.

7. Baughman RP, Sparkman BK, Lower EE. Six-minute walk test and health status assessment in sarcoidosis. Chest. 2007;132:207-213.

8. Baughman RP, Culver DA, Cordova FC, et al. Bosentan for sarcoidosis- associated pulmonary hypertension: a double-blind placebo controlled randomized trial. Chest 2014;145:810-817. 
9. Barnett CF, Bonura EJ, Nathan SD, et al. Treatment of sarcoidosis-associated pulmonary hypertension. A two-center experience. Chest. 2009;135(6):1455-1461.

10. Palmero V, Sulica R. Bosentan for the treatment of sarcoidosisassociated pulmonary hypertension. Am J Respir Crit Care Med 2011;183:A5889. Available at: https://doi.org/10.1164/ ajrccm-conference.2011.183.1_MeetingAbstracts.A5889. Last accessed: July 6, 2020.

11. Qua D, Palmero V, Sulica R. Long-term bosentan therapy improves exercise capacity and hemodynamics in sarcoidosisassociated pulmonary hypertension. Eur Respir J 2012;40(suppl 56):P942. Available at: https://erj.ersjournals.com/content/40/ Suppl_56/P942.short. Last accessed: July 6, 2020.

12. Keir GJ, Walsh SL, Gatzoulis MA, et al. Treatment of sarcoidosis-associated pulmonary hypertension: a single centre retrospective experience using targeted therapies. Sarcoidosis, Vasculitis, and Diffuse Lung Diseases 2014;31(2):82-90.

13. Milman N, Burton CM, Iversen M, et al. Pulmonary Hypertension in end-stage pulmonary sarcoidosis: therapeutic effect of sildenafil? J Heart Lung Transpl. 2008;27(3):329-334.

14. Boucly A, Cottin V, Nunes H, et al. Management and long-term outcomes of sarcoidosis-associated pulmonary hypertension. Eur Respir J 2017;50(4):1700465.

15. Hassoun PM, Zamanian RT, Damico R, et al. Ambrisentan and tadalafil up-front combination therapy in sclerodermaassociated pulmonary arterial hypertension. Am J Respir Crit Care Med. 2015;192(9):1102-1110.

16. Badesch DB, Feldman J, Keogh A, et al, for the ARIES-3 Study Group. ARIES-3: ambrisentan therapy in a diverse population of patients with pulmonary hypertension. Cardiovasc Ther. 2012 Apr;30(2):93-9.

17. Bhandari M, Iqbal M, Mannem SR, et al. Pulmonary vasodilator therapy showed clinical improvement in patients with sarcoidosis associated pulmonary hypertension. Am J Respir Crit Care Med 2020;201:A4082. Available at: https:// www.atsjournals.org/doi/abs/10.1164/ajrccmconference.2014.189.1_MeetingAbstracts.A4082. Last accessed: July 6, 2020 .

\section{Matthew Patel}

Medical Student, Royal College of Surgeons In Ireland, Dublin, Ireland,Email: matthewpatel@rcsi.com

\section{Karim Ladak, MD}

Assistant Professor, McMaster University

Department of Medicine, Hamilton, Ontario, Canada

Received: July 6, 2020

Accepted: July 6, 2020

doi: $10.3121 / \mathrm{cmr} \cdot 2020.1600$ 